\title{
A robust method to determine the contact resistance using the van der Pauw set up.
}

\author{
G. González-Díaz ${ }^{1,2}$ *, D. Pastor ${ }^{1,2}$, E. García-Hemme ${ }^{1,2}$, D. Montero ${ }^{1,2}$, R. García- \\ Hernansanz $^{1,2}$, J. Olea ${ }^{1,2}$, A. del Prado ${ }^{1,2}$, E. San Andrés ${ }^{1,2}$ and I. Mártil ${ }^{1,2}$ \\ ${ }^{1}$ Dept. de Física Aplicada III (Electricidad y Electrónica), Univ. Complutense de Madrid, \\ 28040 Madrid, Spain \\ ${ }^{2}$ CEI Campus Moncloa, UCM-UPM, 28040 Madrid, Spain \\ * Author for correspondence germang@ucm.es
}

\begin{abstract}
The van der Pauw method to calculate the sheet resistance and the mobility of a semiconductor is a pervasive technique both in the microelectronics industry and in the condensed matter science field. There are hundreds of papers dealing with the influence of the contact size, nonuniformities and other second order effects. In this paper we will develop a simple methodology to evaluate the error produced by finite size contacts, detect the presence of contact resistance, calculate it for each contact, and determine the linear or rectifying behavior of the contact. We will also calculate the errors produced by the use of voltmeters with finite input resistance in relation with the sample sheet resistance.
\end{abstract}

\section{1.- Introduction}

The four-point probe measuring technique is very well known from the beginning of the $X X$ century when Wenner proposed it to measure the earth resistivity ${ }^{1}$. Later Valdes ${ }^{2}$ adapted it for semiconductor measurements. The collinear four-point probe has been of great importance for the microelectronic industry. The determination of implantation uniformity over the whole wafer is an example.

The method undergoes an important change with the famous paper by van der Pauw ${ }^{3}$ where the author solved the problem of measurements on arbitrary samples by placing four infinitely small contacts on the sample border. Nevertheless, from a theoretical point of view an ideal point contact has infinite resistance, thus to conduct a finite current requires an infinite applied potential and from the experimental point of view it is impossible to place this kind of contact on a sample. For these reasons it is possible to find a great quantity of papers and books 4,5 dealing with the influence of the contact size on the measurement accuracy. Some of the papers address the subject from a mathematical point of view ${ }^{6}$ while others use experimental set-ups. ${ }^{7}$ The influence of anisotropy ${ }^{8}$ or non-uniformities ${ }^{9}$ is also a point of concern. Some authors face the problem of inhomogeneities using more than four contacts as for example Szymański et al 
${ }^{10}$ which add 2 extra contacts over the usual 4 . Also, the sample thickness has been studied regarding the precision of the measurements ${ }^{11}$.

The main advantage of the collinear four-point probe or the van der Pauw set up is the theoretical independence of the results from the contact resistance. Of course, this insensitivity has limits, some of them related to the sample, as for example, surface currents and others related to the measurement equipment, such as the compliance range of the current source or the impedance and sensitivity of the voltmeters.

In samples where it is suspected the existence of contact resistance it is very usual to use the technique known as Transfer Length Method (TLM) 4, 5, 12 to measure it and also to find the sample resistance. This technique implies defining several contacts, usually by lithography. The main problem is that the sample can not be used for any other purpose, as for example Hall mobility measurements. Having a unique sample to measure the sheet resistance, contact resistance and Hall mobility the method we propose here could be very useful.

Surprisingly, there is not, to our knowledge, any paper dealing with a method to calculate the contact resistance, using the van der Pauw configuration, in spite of the fact that could be easily calculated. We will propose in this paper a methodology to detect and to measure the specific contact resistance for each one of the four contacts.

All through the paper, we will deal with a square sample with contacts at all the corners, uniform in resistivity and thin enough to assure that the voltage equipotentials are bidimensional. Also, we will neglect the thermogalvanic effects that could appear at the contacts. We will assume uniform temperature all over the sample.

\section{2.- Equipment}

The most common set up for van der Pauw measurements uses a constant current source and a sensitive voltmeter. This set-up has a serious drawback because the current source or the voltmeter has to have floating ground. The absence of a common ground is very prone to electronic noise. Some years ago ${ }^{13}$ it was proposed that to improve the signal to noise ratio it would be a better option to use grounded impedance adapters previous to a differential voltmeter or to an ungrounded voltmeter. With the increasing precision and sensitivity of the present analog to digital converter technology, the best option is to use high precision, high impedance digital voltmeters and subtract the readings. Our set-up uses a Keithley SCS-4200 with four SMUs (Source and Measure Unit) sharing a common ground. With this set-up we can measure voltages of 2 volts with a nominal error of $10 \mu \mathrm{V}$. Under the SCS-4200 computerized 
control we can rotate the sample connections and also invert the current source automatically without the use of switches or relays which are other possible sources of errors.

The most common sample shape used in the semiconductor field is a square for its simplicity to be cleaved or defined with lithography. The schematic set-up is depicted on Fig 1. Contact 1 is grounded by setting the SMU to 0 volts. Contact 2 is fed with a constant current and besides we measure the potential developed by the current source. The other two SMUs (contacts 3 and 4) are used just as high impedance voltmeters and we save the voltages of both SMUs referred to ground.

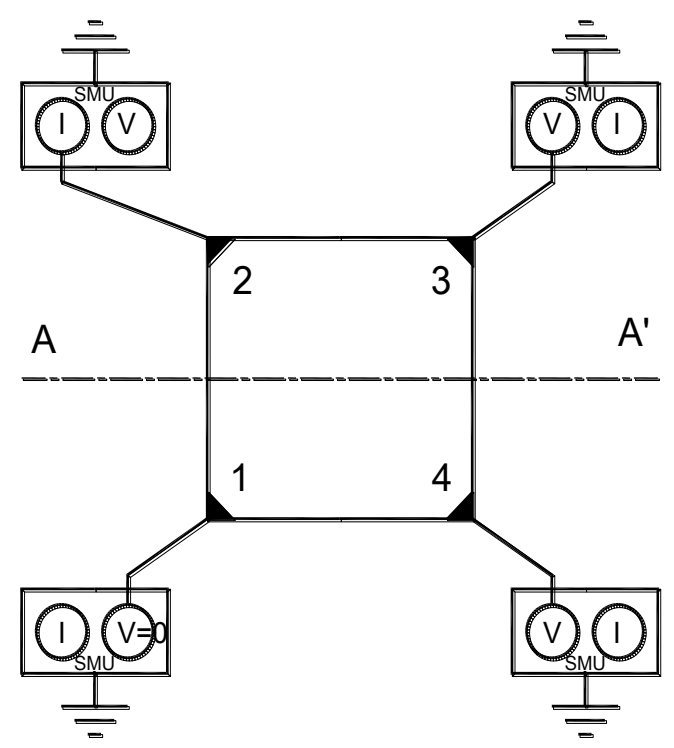

Figure 1: set-up for van der Pauw measurement. The sample is square with triangular contacts at the corners. There is a SMU connected at each corner.

\section{3.- Method to detect the error due to finite size contacts}

The key question in our measurement method is to know the relation between the potentials at the three corners (the potential at the output of the current source, $v_{2}$, and the potentials at the voltage corners, $\mathrm{V}_{3}$ and $\mathrm{V}_{4}$ ). This relation is not analytical and it strongly depends on the contact size, or more precisely the contact size/sample size ratio. Van der Pauw suggested that contact size should be sizeless. In that case, the resistance of a conductor with an infinitely small section is infinite and consequently we will need an infinite potential to force a finite current through this contact i.e. the current source compliance range should be infinite. As the potential difference between contact 3 and contact 4 is not dependent on the voltage on 
contact 2 but exclusively on the injected current, it is clear that the voltage relations are geometry dependent.

As the contact becomes real, with a finite area, the sample resistance observed between corners 1 and 2 becomes smaller, and consequently the current source needs less voltage to introduce the current. However, it is well-known that finite area contacts imply errors when we use the van der Pauw equations ${ }^{3}$.

\section{1.- Description of the method}

To obtain the relations between the potentials at the different corners we have modelled the sample with the PSPICE ${ }^{14}$ circuit simulation software. Among the different PSPICE distributors we have chosen LTspice IV from Linear Technology because it is free, non-limited and more precise than other distributions. In the counter balance the graphics interface is worse than other distributors as Cadence ${ }^{15}$. Other simulation programs like TCAD from Silvaco ${ }^{16}$ can be used with similar results. In PSPICE we define a square mesh of resistors to simulate the square sample under measurement. The mesh is composed of identical resistors, except for those in the outer limits, which have twice the resistance ${ }^{17}$. We force a current between two contiguous contacts, obtaining the potentials in all the nodes. In the upper part of figure 2 , we represent in a 3 dimensional plot the voltage in the vertical axis versus the geometrical $\mathrm{X}$ and $\mathrm{Y}$ coordinates for the case of a mesh of $20 \times 201 \mathrm{~K} \Omega$ resistors fed with a $1 \mathrm{~mA}$ current. The total number of resistors is $2 n(n+1)$, i.e. in our present case 840 resistors. Numbers in the $X$ and $Y$ axis from 1 to 21 correspond to the rows and columns. Of course, the use of this scheme assumes that the interfaces between the sample and the contacts are ohmic, i.e. there is not any current limitation or voltage drop at the interfaces. 

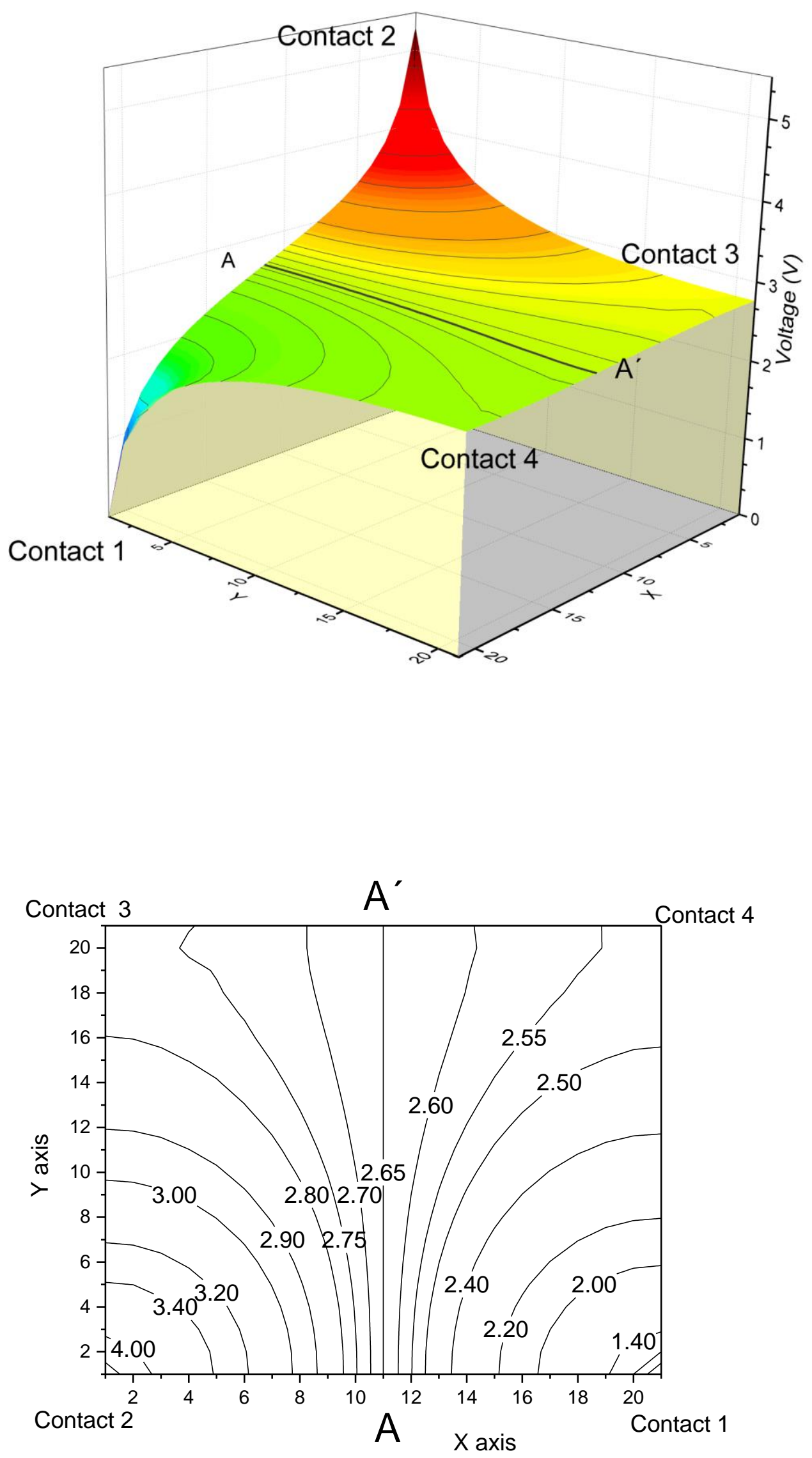

Figure 2: Upper part: $3 \mathrm{D}$ view of the potential distribution on the layer. Vertical axis is the potential. Lower part: $2 \mathrm{D}$ view of the same image. $\mathrm{X}$ and $\mathrm{Y}$ are the geometrical coordinates and $\mathrm{A}-\mathrm{A}^{\prime}$ the symmetry axis 
On the lower part of fig 2 we represent a plane view of the potential, showing the equipotential lines. The line $A A^{\prime}$ (also depicted in fig 1 ) is equipotential with a potential $V_{2} / 2$. As it can be seen in Fig. 2, the potential distribution has symmetry through the axis $A A^{\prime}$.

According to that symmetry, and for a better understanding of the pictures, in the following we will represent just one half of the voltage plots i.e. assuming a 0 potential at line $A A^{\prime}$. We will use this line as the $Y$-axis, and the line that passes through contact 1 and contact 2 will be the $x$ axis. With this Cartesian coordinate system we can write: $V(x, y)=-V(-x, y)$.
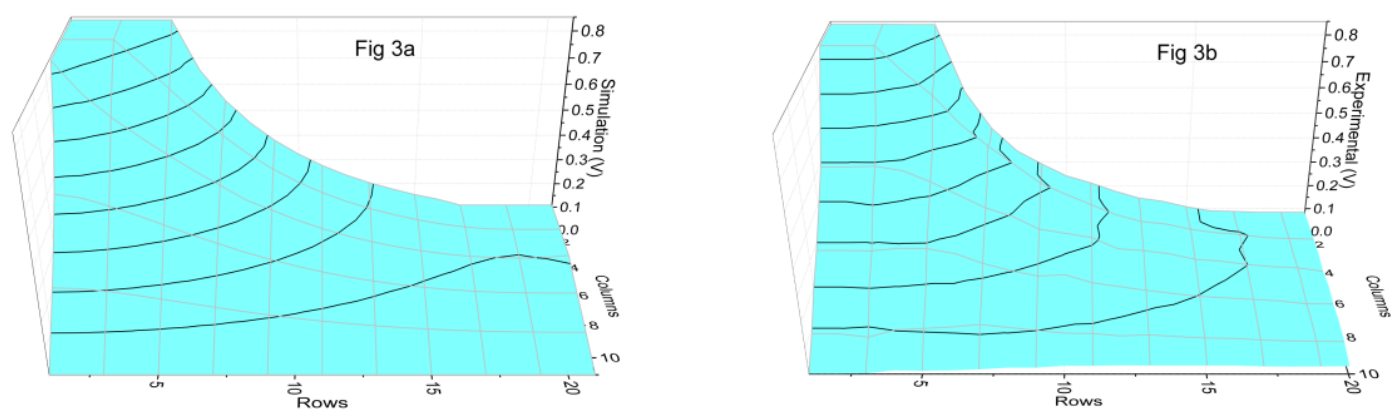

Figure 3: a) potential distribution in a mesh of $20 \times 20$ resistors with triangular contacts. b) experimental values of the potential in a silicon sample $1 \times 1 \mathrm{~cm}$ and triangular contacts

\section{2.- Method validation}

To check the validity of the PSPICE simulation we will compare, at first, the equipotential curves in the model with the same curves in a real sample. To this purpose we have cut a $1 \times 1 \mathrm{~cm}$ silicon sample of a $\mathrm{n}$ type wafer of $5 \pm 2 \Omega \mathrm{cm}$ resistivity and $300 \pm 25 \mu \mathrm{m}$ thickness. We have electron beam evaporated triangular contacts at the corners consisting in $100 \mathrm{~nm} \mathrm{Ti}$ and $200 \mathrm{~nm}$ Al. The triangles are isosceles, having $2 \mathrm{~mm}$ cathetus. We have introduced $10 \mathrm{~mA}$ through contact 2 and contact 1 (ground) and we have used a Karl Suss probe station

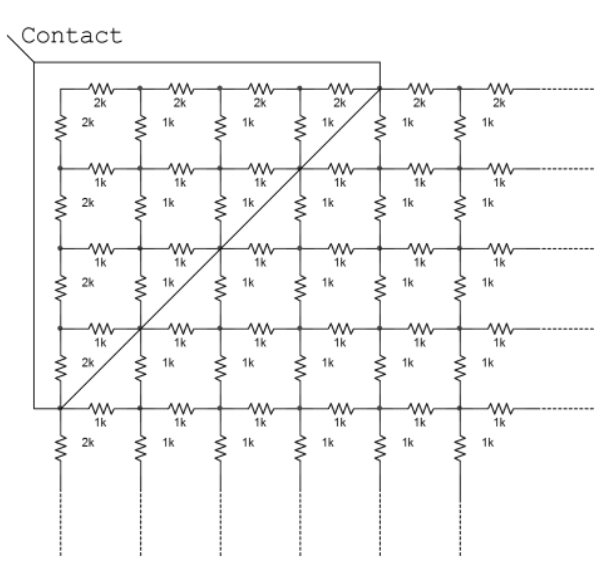

Figure 4: A triangular contact representation shorting 4 resistors in the horizontal and vertical directions with "Everbeing" precision micromanipulators to measure the potential all over the sample in a $500 \mu \mathrm{m}$ mesh. Figure $3 a$ present the simulated equipotential lines obtained with a $20 \times 20$ resistor mesh with $2 \mathrm{~mm}$ triangular contacts and Figure 
$3 \mathrm{~b}$ the experimental measurements. To model the triangular contacts, we have short-circuited 4 resistors in each corner and all the resistors included inside the triangle defined by the hypotenuse, as represented in Fig. 4. The $Z$ axis of the figure $3 \mathrm{~b}$ has been scaled to compare with the simulation results. These plots show almost equal equipotential lines for both figures. The main differences are due to geometrical errors in the contact area and also in the size of the sides of the square sample.

$$
\text { In a classical reference }{ }^{18}, \mathrm{R} \text {. }
$$

Chwang gives some experimental results (also cited by D. Look in reference 5), obtained with an electrolytic cube with square geometry and different electrode sizes, both triangular and square. He uses the quotient between the lateral size of the contact $(\delta)$ and the sample

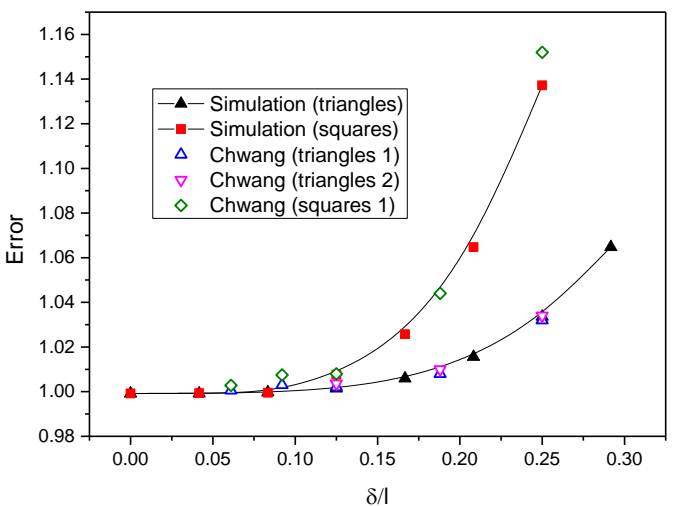

Figure 5: experimental data obtained by Chwang (hollow symbols) and PSPICE simulated data (solid symbols) quotient between the theoretical sheet resistance and the observed sheet resistance as the error measurement represented in the $Y$ axis. In the paper, Chwang compare this experimental results with a model also based on a mesh of resistors but the solution is by far much more complex than the one obtained by using PSPICE. In figure 5 we present the experimental data obtained by Chwang and the ones obtained by using our simulation. There are two sets of experimental values for triangular contacts represented in figure 5 as triangles and inverted triangles and only a set of results with square contacts represented in the picture as squares. Lines with solid squares and solid triangles are the results of our simulation using a mesh of $48 \times 48$ i.e. 4704 resistors. Such a big number of resistors is needed to obtain enough precision to compare our simulated results with the data obtained by Chwang. As it can be seen in the figure, in both cases (triangular and square contacts) the data fit accurately.

\section{3.- Some results with different electrode sizes}

PSPICE allows us to determine the error as a function of the electrode size for a particular shape. In the case of triangular contacts, we represent in table 1 the results for different electrode sizes 
and $1 \mathrm{~mA}$ current. The same for square contacts is represented in table 2 . All cases we are simulating a $48 \times 481 \mathrm{~K} \Omega$ resistor mesh.

The first column is the number of rows and columns that have been short circuited to simulate the contact size, the second column is the percentage of the lateral electrode size $(\delta)$ related to the side of the square sample (I) i.e. in this case the number in the first column divided by 48 and multiplied by 100 . The third column is the voltage developed the current source contact $\left(V_{2}\right)$, fourth and fifth columns are the voltages at the opposite corners $\left(V_{3}\right.$ and $\left.V_{4}\right)$ being the sixth the difference between both. The seventh column is the simulated sheet resistance according to the simulated voltage values of the previous columns and the van der Pauw formula, and the eighth the error respective to its theoretical value. The ninth, $\mathrm{Rp}$, is the resistance seen from the current source to ground (that is to say $V_{2}$ divided by the injected current) which is, by symmetry, the same as the one seen between any two contiguous contacts. The tenth column is the alpha coefficient $(\alpha)$, defined as the quotient between the resistance $R_{p}$ seen between two contiguous corners and the simulated sheet resistance calculated in column 7. Finally, the eleventh column shows $V_{2} / \Delta V$, the quotient between the voltage at the current source and the differential voltage at the opposite corners $\Delta \mathrm{V}=\left(\mathrm{V}_{3}-\mathrm{V}_{4}\right)$.

Table I: Pspice simulation results for a square sample with triangular contacts. See the text for explanation.

\begin{tabular}{|c|c|c|c|c|c|c|c|c|c|c|}
\hline 1 & 2 & 3 & 4 & 5 & 6 & 7 & 8 & 9 & 10 & 11 \\
\hline № & $\delta / I(\%)$ & $\mathrm{V}_{2}(\mathrm{~V})$ & $V_{3}(V)$ & $\mathrm{V}_{4}(\mathrm{~V})$ & $\Delta \mathrm{V}(\mathrm{V})$ & $\operatorname{Sim} . \operatorname{Rs}(\Omega / \square)$ & |error| \% & $\operatorname{Rp}(\Omega)$ & $\alpha$ & $\mathrm{V}_{2} / \Delta \mathrm{V}$ \\
\hline 0 & 0 & 6.42221 & 3.32151 & 3.10070 & 0.22081 & 1000.79 & 0.079 & 6422.21 & 6.41714 & 29.0848 \\
\hline 1 & 2.08 & 4.42221 & 2.32151 & 2.10070 & 0.22081 & 1000.79 & 0.079 & 4422.21 & 4.41872 & 20.0272 \\
\hline 2 & 4.17 & 3.63899 & 1.92990 & 1.70909 & 0.22081 & 1000.79 & 0.079 & 3638.99 & 3.63612 & 16.4802 \\
\hline 4 & 8.33 & 2.79919 & 1.50995 & 1.28924 & 0.22071 & 1000.33 & 0.033 & 2799.19 & 2.79825 & 12.6827 \\
\hline 6 & 12.50 & 2.29447 & 1.25740 & 1.03707 & 0.22033 & 998.61 & 0.138 & 2294.47 & 2.29765 & 10.4138 \\
\hline 8 & 16.67 & 1.93206 & 1.07570 & 0.85636 & 0.21934 & 994.12 & 0.587 & 1932.06 & 1.94347 & 8.80852 \\
\hline 10 & 20.83 & 1.64769 & 0.93274 & 0.71522 & 0.21725 & 984.65 & 1.535 & 1647.69 & 1.67129 & 7.58489 \\
\hline 12 & 25.00 & 1.41149 & 0.812477 & 0.599012 & 0.213465 & 967.50 & 3.250 & 1411.49 & 1.45890 & 6.61228 \\
\hline 14 & 29.17 & 1.20659 & 0.706904 & 0.499685 & 0.207219 & 939.19 & 6.081 & 1206.59 & 1.28471 & 5.82278 \\
\hline
\end{tabular}

Table II: Pspice simulation results for a square sample with square contacts. The meaning of the columns is identical to the ones in Table I

\begin{tabular}{|c|c|c|c|c|c|c|c|c|c|c|}
\hline 1 & 2 & 3 & 4 & 5 & 6 & 7 & 8 & 9 & 10 & 11 \\
\hline № & $\delta / I(\%)$ & $\mathrm{V}_{2}(\mathrm{~V})$ & $\mathrm{V}_{3}(\mathrm{~V})$ & $\mathrm{V}_{4}(\mathrm{~V})$ & $\Delta \mathrm{V}(\mathrm{V})$ & $\operatorname{Sim} . \operatorname{Rs}(\Omega / \square)$ & |error| \% & $\operatorname{Rp}(\Omega)$ & $\alpha$ & $\mathrm{V}_{2} / \Delta \mathrm{V}$ \\
\hline 0 & 0 & 6.42221 & 3.32151 & 3.10070 & 0.22081 & 1000.79 & 0.079 & 6422.21 & 6.41713 & 29.0847 \\
\hline 1 & 2.08 & 4.04448 & 2.13264 & 1.91184 & 0.22080 & 1000.75 & 0.075 & 4044.48 & 4.04146 & 18.3173 \\
\hline
\end{tabular}




\begin{tabular}{|l|l|l|l|l|l|l|l|l|l|l|}
\hline 2 & 4.17 & 3.22519 & 1.72299 & 1.50220 & 0.22079 & 1000.69 & 0.069 & 3225.19 & 3.22293 & 14.6075 \\
\hline 4 & 8.33 & 2.36906 & 1.29490 & 1.07415 & 0.22074 & 1000.48 & 0.048 & 2369.06 & 2.36783 & 10.7318 \\
\hline 6 & 12.50 & 1.85964 & 1.03929 & 0.82034 & 0.21895 & 992.401 & 0.7598 & 1859.64 & 1.87395 & 8.49344 \\
\hline 8 & 16.67 & 1.49497 & 0.85503 & 0.63993 & 0.21510 & 974.928 & 2.5071 & 1494.97 & 1.53344 & 6.95011 \\
\hline 10 & 20.83 & 1.20917 & 0.70819 & 0.50097 & 0.20722 & 939.218 & 6.0781 & 1209.17 & 1.28745 & 5.83519 \\
\hline 12 & 25.00 & 0.97187 & 0.58295 & 0.38892 & 0.19402 & 879.382 & 12.061 & 971.877 & 1.10513 & 5.00886 \\
\hline
\end{tabular}

The last two columns are related through the factor $\pi / \ln (2)$ because according to van der Pauw:

$$
\begin{aligned}
& R_{S}=\frac{\pi}{\ln (2)} \frac{V_{3}-V_{4}}{I}=\frac{\pi}{\ln (2)} \frac{\Delta V}{V_{2}} R_{p} \\
& \frac{V_{2}}{\Delta V}=\frac{R_{p}}{R_{s}} \frac{\pi}{\ln (2)}=\frac{\alpha \pi}{\ln (2)}
\end{aligned}
$$

and the aforementioned relation holds. The relation between column 3 and column 9 is the injected current, in this case $1 \mathrm{~mA}$.

From the results on table $I$ it is clear that using triangular contacts on the corners of a square sample is a good choice because even with a cathetus as big as a $25 \%$ of the sample side the error is only about $3 \%$ while with square contacts the error increases to a value as high as $12 \%$. In the samples described up to this point, in which we have assumed there is not any contact resistance, we will have $V_{2}=V_{3}+V_{4}$ irrespective of the contact size. This relation holds from symmetry because $V_{2}=\left(V_{2}-V_{3}\right)+V_{3}$ and $\left(V_{2}-V_{3}\right)=\left(V_{4}-V_{1}\right)=V_{4}$ as $V_{1}=0$. This relation can be checked in table 1.

The former relation can be extended to any sample with mirror symmetry and equidistant contacts as long as they have the same shape and size. If a particular symmetrical sample with non-limiting and well defined contacts does not follow the equation $V_{2}=V_{3}+V_{4}$ this means that we have some kind of non-uniformity in the sheet resistance.

\section{4.- Contact resistance determination:}

\section{1- Theory}

While in theory the van der Pauw technique is not prone to errors due to the presence of contact resistance, the fact is that above a determined resistance added by the contacts, the measurements become noisy and erratic. Consequently it is interesting to have a procedure to know:

1.- if there is an important contact resistance,

2.- if the contact resistance is the same in the four contacts 
3.- if any or all of them are rectifying.

Of course the contact resistance depends on the contact area. To obtain the specific contact resistance we have to divide the contact resistance by the contact area. Those contact resistances are not the resistances between the probes and the metal contact, which is usually negligible, but the contact resistance between the evaporated metal and the sample. In the case of semiconductors those resistances could be appreciable and depend on the current direction and the current value themselves because they are frequently non-ohmic. It is well known that in case of III-V or II-VI semiconductors the issue of making good contacts could be a real problem. In the paper we give the method to detect the contact non linearity and its dependence on the current.

Figure 6 shows the potentials in a sample with contact resistances $\left(R_{c}\right)$. In this figure we distinguish between the measured potentials at the metallic electrodes $V_{i}$ (extrinsic voltages) and the potentials in the corners of the sample $\mathrm{V}^{\prime}{ }_{\mathrm{i}}$ (intrinsic voltages)

The process to determine the contact resistance starts by calculating the $\alpha$ parameter. It is supposed that we know the geometry of our sample and the relative size of the contacts. From these data we can generate with PSPICE a table similar to table 1 for a particular set of sample and contact size and shape. In the case represented in figure $6, \alpha \pi / \ln (2)=\left(\mathrm{V}_{2}{ }_{2}-\mathrm{V}^{\prime}{ }_{1}\right) /\left(\mathrm{V}^{\prime}{ }_{3}-\mathrm{V}^{\prime}{ }_{4}\right)=$ $\left(V^{\prime}{ }_{2}-V^{\prime}{ }_{1}\right) /\left(V_{3}-V_{4}\right)$ because $V^{\prime}{ }_{3}=V_{3}$ and $V^{\prime}{ }_{4}=V_{4}$ as we assumed that all the voltmeters and specially those connected in corners 3 and 4 have an almost infinite impedance, and consequently there is not current through $R_{c 3}$ and $R_{c 4}$

Having the differential voltage $\left(V_{3}-V_{4}\right)$ and the table we can obtain $\left(V^{\prime}{ }_{2}-V^{\prime}{ }_{1}\right)$. The difference between the voltage at corner $2\left(V_{2}\right)$ and $\left(V^{\prime}{ }_{2}-V^{\prime}{ }_{1}\right)$ gives us the voltage drop at the current source contacts. Of course, if we divide this voltage drop by the current injected we will obtain the contact resistance of the two contacts in series:

$$
\left(R_{c 2}+R_{c 1}\right)=\frac{V_{2}-\left(V^{\prime}{ }_{2}-V^{\prime}{ }_{1}\right)}{I}=\frac{V_{2}-\left(V_{3}-V_{4}\right) \cdot\left(\frac{\alpha \pi}{\ln (2)}\right)}{I}
$$




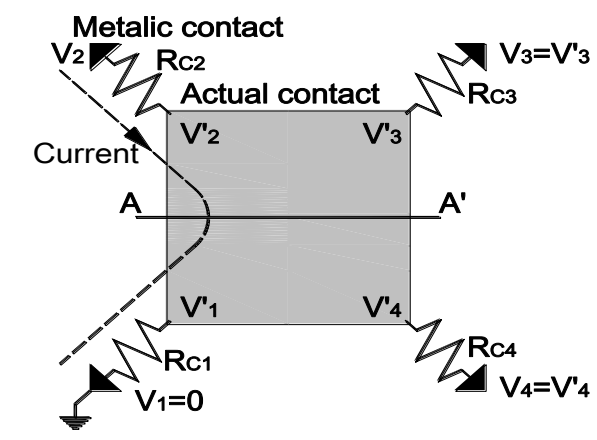

Figure 6: potential distribution in a sample with contact resistance. Black triangles are the metallic contacts.
The same result could be found if we measure, in addition, the potentials at $V_{2}$ and $V_{1}$ while we inject current at contact $\mathrm{V}_{3}$ and contact $\mathrm{V}_{4}$ is connected to ground. To differentiate both rotations we will call $V_{2}$ the potential at corner 2 when the current source is connected at this contact, and V" ${ }_{2}$ and $V^{\prime \prime}{ }_{1}$ when the current source is connected to contact 3 . It is

straightforward that

$$
R_{C 1}+R_{C 2}=\frac{V_{2}-\left(V_{2}^{\prime \prime}-V_{1}^{\prime \prime}\right)}{I}
$$

To distinguish between $R_{c 1}$ and $R_{c 2}$ we have to bear in mind that the line $A A^{\prime}$ is equipotential, irrespective of the contact resistances. Its potential is $\left(V_{3}+V_{4}\right) / 2$ or $\left(V^{\prime}{ }_{3}+V^{\prime}{ }_{4}\right) / 2$. Consequently we can write:

$$
\begin{aligned}
& V^{\prime}{ }_{2}=\frac{V_{3}+V_{4}}{2}+\frac{V_{3}-V_{4}}{2}\left(\frac{\alpha \pi}{\ln (2)}\right) \\
& V^{\prime}{ }_{1}=\frac{V_{3}+V_{4}}{2}-\frac{V_{3}-V_{4}}{2}\left(\frac{\alpha \pi}{\ln (2)}\right)
\end{aligned}
$$

and the contact resistances could be easily deduced as follows:

$$
\begin{aligned}
& R_{c 2}=\frac{V_{2}-V^{\prime}{ }_{2}}{I}=\frac{1}{I}\left(V_{2}-\frac{V_{3}+V_{4}}{2}-\frac{V_{3}-V_{4}}{2}\left(\frac{\alpha \pi}{\ln (2)}\right)\right) \\
& R_{c 1}=\frac{V^{\prime}{ }_{1}}{I}=\frac{1}{I}\left(\frac{V_{3}+V_{4}}{2}-\frac{V_{3}-V_{4}}{2}\left(\frac{\alpha \pi}{\ln (2)}\right)\right)
\end{aligned}
$$

These equations give us the contact resistances at the current source corners. By reversing the current, the rectifying character of the contacts could be detected. Also the linearity could be checked by changing the current source value. From equations 6 and 7 it is easy to prove that the condition $V_{2}=V_{3}+V_{4}$ also holds if $R_{c 1}=R_{c 2}$. Finally, to evaluate $R_{c 3}$ and $R_{c 4}$ we can apply the same procedure, but injecting the current through contact 3 and 4 .

To summarize all the former ideas we present in Fig 7 a flowchart of a complete process of measurement of the sheet resistance. It is assumed that the film under test is a single layer i.e. that it is homogeneous in depth, thin enough to have a two dimensional potential distribution 
and that the sample geometry is precise enough. The process starts by calculating the $\alpha$ parameter associated with the particular geometry we have. Then we have to measure for each contact rotation the three potentials $V_{i}$ for a particular injected current. For each configuration we have to check the equality $\frac{a \pi}{\ln (2)}\left(V_{3}-V_{4}\right)=V_{2}$. If it is verified for the four sample rotations, we can guarantee that there are not any relevant contact resistances at any of the four corners. Now, the $V_{i}$ s are equal to $V_{i}^{\prime}$ s and we can check the in plane film uniformity by using the relation $V_{2}=V_{3}+V_{4}$. If the equality does not hold, at least for a sample rotation, it means that the film is not uniform and the sheet resistance calculation is not reliable. If the equation holds, it is an indicator that the sheet resistance calculated as an average is a precise value. Using the $\alpha$ parameter we can estimate the error in the sheet resistance and eventually correct it.

If $\frac{a \pi}{\ln (2)}\left(V_{3}-V_{4}\right)=V_{2}$ does not hold, we can use equations [6] and [7] to calculate the contact resistances and deduce the intrinsic potentials $V^{\prime}{ }_{i}$ for the four corners. Having these potentials we can follow the procedure explained above.

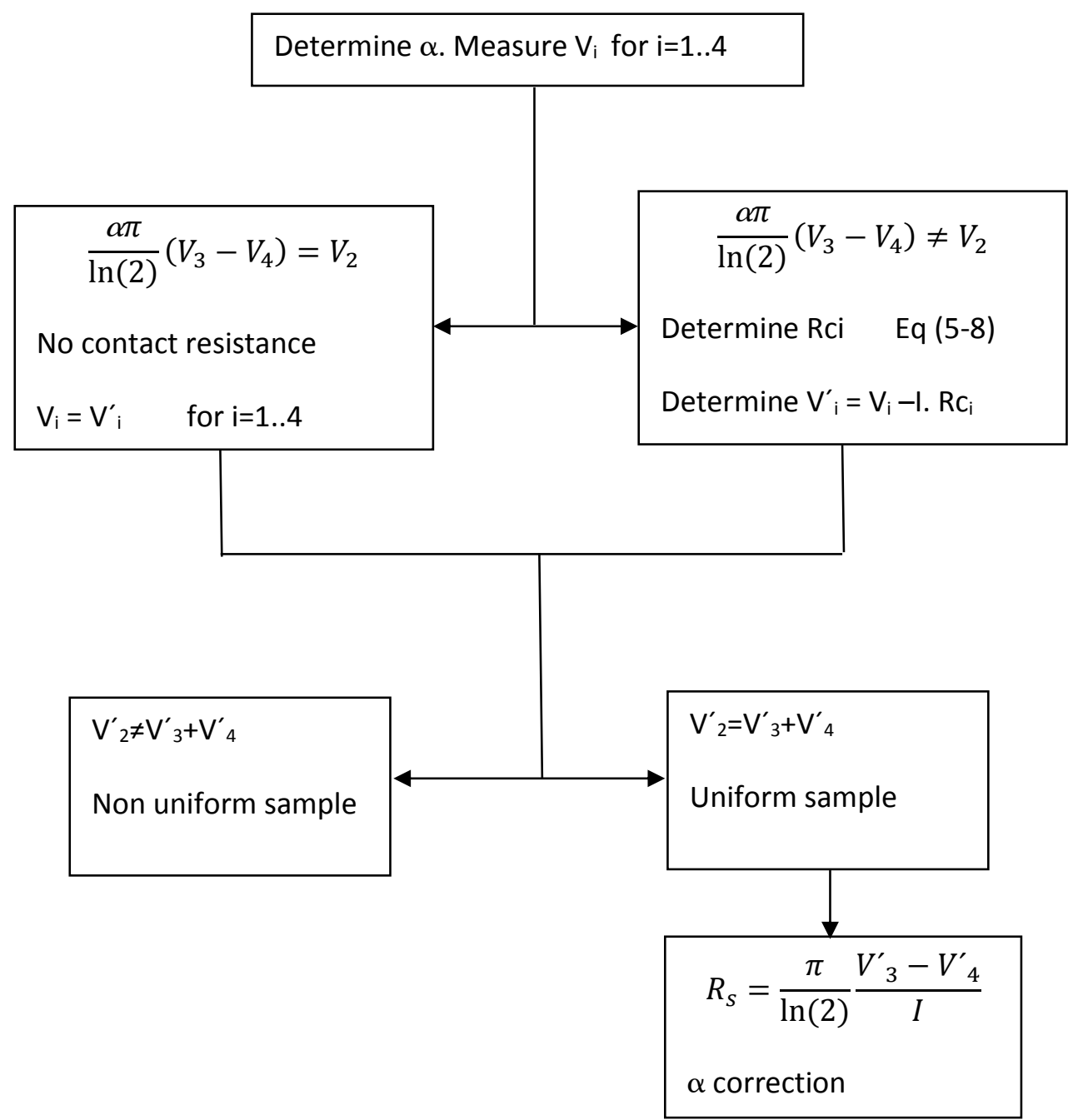


Fig 7: flowchart to determine the sheet resistance, the contact resistance and the validity of a sheet resistance measurement

\section{2.- Experiment}

To check the validity of the previous theory we present in Table III some measurements made on a $1 \times 1 \mathrm{~cm}$ silicon sample with evaporated aluminum triangular electrodes having a $\delta / /$ ratio of $0.15 \pm 0.02$. The sample thickness is $0.3 \mathrm{~mm}$ and the approximate resistivity $200 \Omega . \mathrm{cm}$. According to table I the alpha parameter has to be from $1.896-2.224$ with a nominal value of 2.048 . The measurements were taken by rotating the sample, and each row represents a 90 degree rotation. The current injected is $1 \mathrm{~mA}$. Column 1 is the voltage measured at the current source, column 2 is the ground connected electrode, columns 3 and 4 are the voltages at measurement corners. Column 5 is the calculated sheet resistance including the error due to the uncertainty of the $\delta / /$ ratio. Values of the contact resistances are calculated at columns 6 and 7 , taking also into account the errors due to the imprecision in the contact sizes.

Table III. See text for explanation

\begin{tabular}{|l|l|l|l|l|l|l|}
\hline $\begin{array}{l}\text { Power supply } \\
\text { (V) }\end{array}$ & Ground & \multicolumn{2}{|l|}{$\begin{array}{l}\text { Voltage at measurement } \\
\text { corners }(\mathrm{V})\end{array}$} & $\begin{array}{l}\text { Sheet Resis- } \\
\text { tance }(\mathrm{K} \Omega / \square)\end{array}$ & $\begin{array}{l}\text { Contact resistances } \\
\text { (from power supply } \\
\text { to sample) }(\mathrm{K} \Omega)\end{array}$ & $\begin{array}{l}\text { Contact resistances } \\
\text { (from sample to } \\
\text { ground) }(\Omega)\end{array}$ \\
\hline $\mathrm{V} 2=20,5203$ & $\mathrm{~V} 1$ & $\mathrm{~V} 3=7,1669$ & $\mathrm{~V} 4=5,7143$ & $6.610 \pm 0.015$ & $\mathrm{Rc} 2=7.33 \pm 0.57$ & $\mathrm{Rc} 1=(-3.0 \pm 5.6) \times 10^{2}$ \\
\hline $\mathrm{V} 3=21,7431$ & $\mathrm{~V} 2$ & $\mathrm{~V} 4=8,0985$ & $\mathrm{~V} 1=6,4293$ & $7.596 \pm 0.018$ & $\mathrm{Rc} 3=6.72 \pm 0.57$ & $\mathrm{Rc} 2=(-4.8 \pm 6.6) \times 10^{2}$ \\
\hline $\mathrm{V} 4=31,2227$ & $\mathrm{~V} 3$ & $\mathrm{~V} 1=8,0049$ & $\mathrm{~V} 2=6,5320$ & $6.702 \pm 0.016$ & $\mathrm{Rc} 4=17.11 \pm 0.58$ & $\mathrm{Rc3}=(4.2 \pm 5.8) \times 10^{2}$ \\
\hline $\mathrm{V} 1=24,8892$ & $\mathrm{~V} 4$ & $\mathrm{~V} 2=9,2083$ & $\mathrm{~V} 3=7,4400$ & $8.047 \pm 0.019$ & $\mathrm{Rc} 1=8.35 \pm 0.70$ & $\mathrm{Rc4}=(1.1 \pm 7.0) \times 10^{2}$ \\
\hline
\end{tabular}

A first glance to the table shows that the sample probably is not perfectly square or the contacts have not the same area because sheet resistance for configuration 1 and 3 is different from resistance in configurations 2 and 4 . As we have 4 rotations and we determine 2 resistances for each rotation we have 8 resistance values corresponding to the 4 resistances, but measured in the two current directions.

Clearly when the current is entering the sample the contact resistance is in the order of kiloohms, while when the current is going out of the sample the resistance is below the error produced by the imprecision of the contact area. That points to rectifying contacts in all the corners. A better lithography would have been necessary to obtain more precise results. 


\section{5.- Comments about the measurement equipment}

\subsection{Voltmeter sensitivity}

If we had used a differential voltmeter at corners 3 and 4 , the main figure of merit would have been the Common Mode Rejection Ratio, defined as the quotient between the differential gain over the common gain, in decibels.

Assume from now that the contacts are non- limiting. In this case the common voltage i.e. the voltage at the line $A-A^{\prime}$ in the Fig 1 and in the lower part of Fig 2 is $\left(V_{3}+V_{4}\right) / 2$ and the quotient differential mode over common mode is:

$$
\frac{V_{\text {differential }}}{V_{\text {common }}}=\frac{V_{3}-V_{4}}{\left(V_{3}+V_{4}\right) / 2}=\frac{2\left(V_{3}-V_{4}\right)}{V_{2}}=\frac{2 \ln (2)}{\alpha \pi}
$$

irrespective of the sample sheet resistance. Now, and depending on $\alpha$, the differential amplifier CMRR is straightforward.

We are not using a differential voltmeter but two digital voltmeters. In this case the formula [8] gives us the sensitivity we need for the voltmeters connected to contacts 3 and 4 . This value of sensitivity has to be consistent with the $\alpha$ parameter, which in turn determines the geometrical error. For the sake of clarity let us assume a $\delta / I$ value of 0.16 . According to Table $1 \alpha=2$ and the error is $0.58 \%$. In that case the quotient of equation 8 is 0.220 , and if we want to preserve this error level the voltmeters should have a sensitivity of $0.13 \%(0.58 \times 0.220 \%)$ i.e. if the common mode is for instance $1 \mathrm{~V}$ the voltmeter has to be able to detect $1.3 \mathrm{mV}$. This is clearly accomplished with a 4 and a half digit voltmeter and is in the limit of the sensitivity of a 3 and a half digit voltmeter.

The sensitivity required, in the case of ohmic contacts, depends on the alpha parameter and not on the injected current. It could be really demanding for samples with very small contacts relative to the sample size. In the case of limiting contacts, the burden resistance forces the use of more sensitive voltmeters as the common mode increases substantially. In that case the differential voltage is the same as in the former case $\left(V_{3}-V_{4}\right)=\left(V^{\prime}{ }_{3}-V^{\prime}{ }_{4}\right)$, but the common mode is increased as:

$$
V_{\text {common }}=I R_{c 1}+\frac{V_{2}^{\prime}-V_{1}^{\prime}}{2}=I R_{c 1}+\frac{V_{3}-V_{4}}{2} \frac{\alpha \pi}{\ln (2)}
$$


Using this expression and the van der Pauw formula for the sheet resistance it is easy to obtain finally:

$$
\frac{V_{\text {diferential }}}{V_{\text {common }}}=\frac{V_{3}-V_{4}}{I R_{c 1}+\frac{V_{3}-V_{4}}{2} \frac{\alpha \pi}{\ln (2)}}=\frac{2 \ln (2)}{\alpha \pi} \frac{1}{\left(1+\frac{2 R_{c 1}}{\alpha R s}\right)}
$$

Now, the voltmeter sensitivity has to be increased over that for the case when the contacts are ohmic in an amount that depends on the quotient between the resistance at contact 1 divided by the sheet resistance and the $\alpha$ parameter.

\section{2.- Voltmeter impedance}

Figure 8 represents the quotient between the resistance seen from two contiguous contacts Rp over the sheet resistance Rs versus the relative size contact $(\delta / I)$ for triangular (triangles) and square contacts (squares) i.e the $\alpha$ parameter. The data for this graph

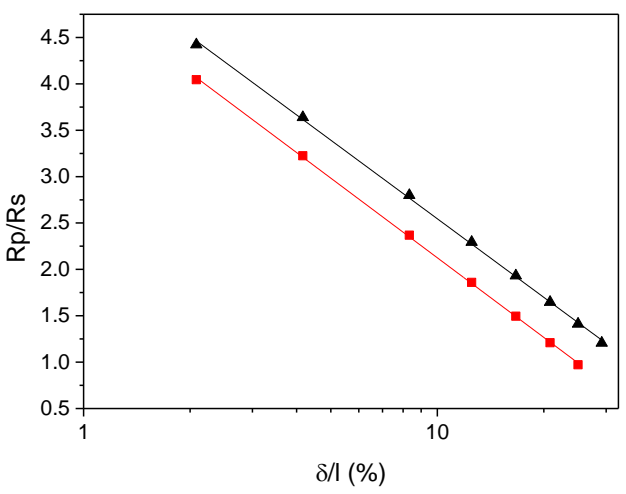

Figure 8: Two contiguous contact resistance over sheet resistance versus the relative size of the contact

in Table I and Table II, except for 0

$\delta / \mathrm{I}$, as it has not physical meaning. As it can be seen, data fit almost perfectly to a logarithmic behavior. The fitting equation could be used to calculate the resistance of the sample between two contiguous contacts if we know the sheet resistance. This value is important because in case the $\delta /$ I value is very low when making high precision measurements, the resistance Rp could be several times the value of the sample sheet resistance and the impedance of the voltmeter used at the corners should be increased accordingly. 
In fact, the resistance Rp for the first row of table I and II should have been infinite. However, for this contact to have an infinitely small area the number of rows and columns in the mesh should have been set as infinite. Looking for an equivalent area for this contact we can solve the fitting equations and we will find a $\delta / l$ value of $0.00419(\delta=0.2)$ for the triangular contacts and 0.0031 $(\delta=0.15)$ for the square contacts.

Most of the books and papers dealing with the Van der Pauw technique give the caution to use a differential

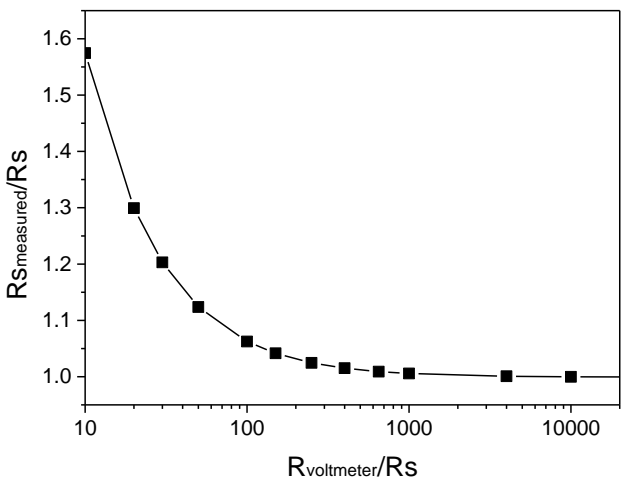

Figure 9: Influence of the voltmeters internal impedance on the measured sheet resistance. $Y$ axis represent the measured sheet resistance over the theoretical one while $X$ axis represent he quotient between the impedance of the voltmeters and the sheet resistance

voltmeter or (as in our case) voltmeters having an input impedance bigger than the sheet resistance or in some cases bigger than the resistance seen between each two corners but without specifying the value of this impedance. Of course, the impedance needed depends strongly on the sheet resistance, the contact size and the error we are ready to accept. In the figure 9 we give the quotient between the simulated sheet resistance over the nominal one in the simulation of a mesh of $48 \times 48$ resistors versus the quotient of the voltmeter impedance over the nominal sheet resistance. We have simulated the voltmeters just as impedances connected at corners 3 and 4 . As it can be seen, to obtain the precision of 3\% (the same precision obtained with $0.25 \delta / I)$ we would need a voltmeter with a resistance about 300 times higher than that of the sheet resistance.

\section{Conclusions:}

In this paper we show that with the correct equipment and a carefully set up design it is possible to obtain a great quantity of information from the van der Pauw measurements that goes beyond the sole determination of resistivity. First of all, the PSPICE simulation gives us the possible errors that could be caused by geometrical factors, in particular the contact size. The acquisition of the voltages on all the corners relative to ground, together with PSPICE data allows us to determine the contact resistance for all the contacts. By varying the value and the direction of the current injected by the source we can also obtain an insight into the linearity and rectifying 
properties of the contacts. Also we can calculate the error produced by the finite voltmeter impedance.

\section{Acknowledgements}

Authors would like to acknowledge the CAI de Técnicas Físicas of the Universidad Complutense de Madrid for the metallic evaporations and sample etching. This work was partially supported by the Project MADRID-PV (Grant No. P2013/MAE-2780) funded by the Comunidad de Madrid and by the Spanish MINECO (Economic and Competitiviness Ministery) under grant TEC 201341730-R

Research by E. García-Hemme was also supported by a PICATA predoctoral fellowship of the Moncloa Campus of International Excellence (UCM-UPM). D. Pastor acknowledges the financial support to the grant EX-2010-0662 from the Spanish Science Ministry

\section{Bibliography}

\footnotetext{
${ }^{1}$ F. Wenner, "A Method of Measuring Earth Resistivity," Bulletin of the Bureau of Standards 12, 469-478, 1915

2 L.B. Valdes, "Resistivity Measurements on Germanium for Transistors," Proc. IRE 42, 420-427, Feb. 1954

${ }^{3}$ L.J. van der Pauw, "A Method of Measuring Specific Resistivity and Hall Effect of Discs of Arbitrary Shape," Phil. Res. Rep. 13, 1-9, Feb. 1958.

${ }^{4}$ D.K. Schroder "Semiconductor Material and Device Characterization". 3th ed. John Wiley and Sons Hoboken NJ 2006

${ }^{5}$ D. Look “Electrical Characterization of GaAs Materials an Devices" John Wiley and Sons 1989

${ }^{6}$ Yicai Sun, Oswin Ehrmann, Jijrgen Wolf, and Herbert Reich "The correction factors and their new curve for the measurement of sheet resistance of a square sample with a square four-point probe" Rev. Sci. Instrum. 63 (7), July 1992

7 Josef Náhlík, Irena Kašpárková, Premysl Fitl “Influence of non-ideal circumferential contacts on errors in the measurements of the resistivity of layers using the van der Pauw method" Measurement 46 (2013) 887-892

${ }^{8}$ O. Bierwagen, * R. Pomraenke, S. Eilers, and W. T. Masselink "Mobility and carrier density in materials with anisotropic conductivity revealed by van der Pauw measurements" Physical Review B 70, 165307 (2004)

${ }^{9}$ Oliver Bierwagen,a Tommy Ive, Chris G. Van de Walle, and James S. Speck "Causes of incorrect carriertype identification in van der Pauw-Hall measurements" Applied Physics Letters 93, 2421082008

${ }^{10}$ Krzysztof Szymański, Kamil Łapiński, Jan L Cieśliński, Artur Kobus,Piotr Zaleski, Maria Biernacka and Krystyna Perzyńska. "Determination of the Riemann modulusand sheet resistivity by a six-point generalization of the van der Pauw method" Meas. Sci. Technol. 26 (2015) 085012

${ }^{11}$ C. Kasla and M. J. R. Hoch "Effects of sample thickness on the van der Pauw technique for resistivity measurements" Review Of Scientific Instruments 76, 033907 s2005d

12 M. Heinrich, S. Kluska, S. Binder, Z. Hameiri, B. Hoex and A.G. Aberle "Accurate potential drop sheet resistance measurement of laser-doped areas in semiconductors" J. Appl. Phys 116, 134505 (2014)

13 "Low level measurements" $6^{\text {th }}$ edition Keithley Instruments Inc. www.keithley.com

${ }^{14}$ http://www.linear.com/designtools/software/

${ }^{15} \mathrm{http}: / /$ www.cadence.com/products/orcad/pages/downloads_verify.aspx

${ }^{16}$ http://www.silvaco.com/
} 
${ }^{17}$ D. W. Koon "Nonlinearity of resistive impurity effects on van der Pauw measurements" Review Of Scientific Instruments 77, 0947032006

${ }^{18}$ R. Chwang, B.J. Smith and C.R. Crowell. "Contact size effects on the van der Pauw method for resistivity and Hall coefficient measurement" Solid State Electronics 17 (1974) 1217-1227 